This item was submitted to Loughborough's Research Repository by the author.

Items in Figshare are protected by copyright, with all rights reserved, unless otherwise indicated.

\title{
On the excitation of a closely spaced array by a line source
}

PLEASE CITE THE PUBLISHED VERSION

LICENCE

CC BY-NC-ND 4.0

\section{REPOSITORY RECORD}

Thompson, lan, and C.M. Linton. 2019. "On the Excitation of a Closely Spaced Array by a Line Source". figshare. https://hdl.handle.net/2134/2289. 


\title{
On the excitation of a closely spaced array by a line source
}

\author{
I Thompson* and C M Linton \\ Department of Mathematical Sciences, Loughborough University, Loughborough, Leics. UK \\ *Email: i.thompson@lboro.ac.uk
}

\begin{abstract}
An infinite row of periodically spaced, identical rigid circular cylinders is excited by an acoustic line source, which is parallel to the generators of the cylinders. A method for calculating the scattered field accurately and efficiently is presented. When the cylinders are sufficiently close together, Rayleigh-Bloch surface waves, which propagate energy to infinity along the array are excited. An expression is derived which enables the amplitudes of these surface waves to be computed without requiring the solution to the full scattering problem.
\end{abstract}

\section{Introduction}

The interaction of waves with periodic arrays is of fundamental importance in many physical contexts. When an infinite array is excited by a plane wave, the theoretical treatment of the problem can be greatly simplified by taking account of the periodicity of the incident wave and of the array. When the excitation is due to a point or line source, however, the incident field is no longer periodic and the resulting problem is more challenging. Nevertheless, there is usually no great difficulty provided the scattered field decays to zero as one moves along the array away from the source. In this article we wish to focus on a situation where this is not the case due to the presence in the scattered field far from the source of Rayleigh-Bloch surface waves travelling along the array. We consider two-dimensional scattering by an infinite row of periodically-spaced, identical circular cylinders excited by a line source which is parallel to the generators of the cylinders. We will refer primarily to the acoustic setting in which we look for time-harmonic solutions $\operatorname{Re}[\phi(x, y) \exp (-\mathrm{i} \omega t)]$ so that the acoustic potential $\phi$ satisfies the two-dimensional Helmholtz equation $\left(\nabla^{2}+k^{2}\right) \phi=0$ in the region exterior to the cylinders, where $k=\omega / c$ and $c$ is the speed of sound. The scatterers are assumed to be rigid and so the normal derivative of $\phi$ must vanish on the cylinder boundaries; the boundary-value problem is thus also applicable to the electromagnetic scattering by an array of perfect conductors of an $S$-polarized incident field.

This geometry has been chosen because it is known that Rayleigh-Bloch surface waves can propagate along such an array $[1],[2],[3]$. These RB waves, as we will henceforth refer to them, decay exponentially away from the array but travel along the array undamped and our main objective is to develop a method by which the amplitude of the RB waves that are excited can be determined without having to solve the full scattering problem. Note that if the boundary condition on the cylinders was changed to $\phi=0$ then no such waves exist [4].

Previous theoretical work on aperiodic excitation of periodic structures, when the structure supports pure surface waves, is scarce. The first attempts to tackle this type of problem 
were made over 40 years ago but little progress appears to have been made since then. For the case of electromagnetic scattering due to a magnetic line current in the presence of a grounded slab covered by an infinite conducting plane containing a periodic array of narrow slots, progress was made in [5] via an approximate sampling technique which reduced the problem to one which could be solved with the aid of Fourier transforms. A different approximate technique, which again reduced the problem to one for which Fourier transforms could be used, was used in [6] to study point source excitation of an array of dipoles. In that paper the question which is or primary concern here was addressed; how can one accurately and efficiently determine the amplitude of the surface wave that is excited?

A general approach to problems in which a periodic structure is excited by an aperiodic incident field is the so-called continuous phased-array or array scanning method in which the solution for aperiodic excitation is written as an integral of the solution for periodic excitation, the integration variable being the phase of the incident field. This approach was used in [7] to analyse line source radiation from a dielectric slab backed by a metal strip grating. The Fourier integral approach used in $\S 4$ is essentially equivalent to the array scanning method. For some recent applications of this technique, see [8, 9].

The paper is organised as follows. The problem is formulated in $\S 2$ and the existence and nature of the surface waves that can propagate along the array is discussed in $\S 3$. In $\S 4$ we present our method which allows the amplitude of the surface waves that are generated to be computed accurately without solving the full scattering problem and in $\S 5$ an analysis of the nature of the far field is given. Numerical results are presented for the amplitudes of the RB waves that are excited and the directional dependence of the overall scattered field. Contour plots of the total field in the presence of a long but finite array are shown which demonstrate that the far field for the infinite array predicts the nature of the field very well. A quantity of particular interest is the proportion of the energy emitted by the source that propagates to infinity along the array and a method for computing this quantity is given in $\S 6$.

\section{General theory}

Consider the scattering of a cylindrical wave by an infinite row of circular cylinders of radius $a<0.5$, located at the points $(x, y)=(p, 0)$ where $p \in \mathbb{Z}$. A line source is positioned at $(X, Y)$, and $\left(R_{p}, \Theta_{p}\right)$ represents the magnitude and orientation of the vector from this point to the centre of cylinder $p$; see figure 1 . The method described below is valid for all source positions, except those at which $Y=0$; numerous subsequent equations involve series and integrals that are divergent when this is the case. Without further loss of generality, we assume that $Y<0$ so that the source is positioned below the array as in figure 1 . The total field is then expressed in the form

$$
\phi^{\mathrm{t}}(x, y ; X ; Y)=\phi^{\mathrm{i}}(x, y ; X ; Y)+\phi^{\mathrm{s}}(x, y ; X ; Y),
$$

where the incident wave and scattered response are given by

$$
\phi^{\mathrm{i}}=\mathrm{H}_{0}(k S),
$$




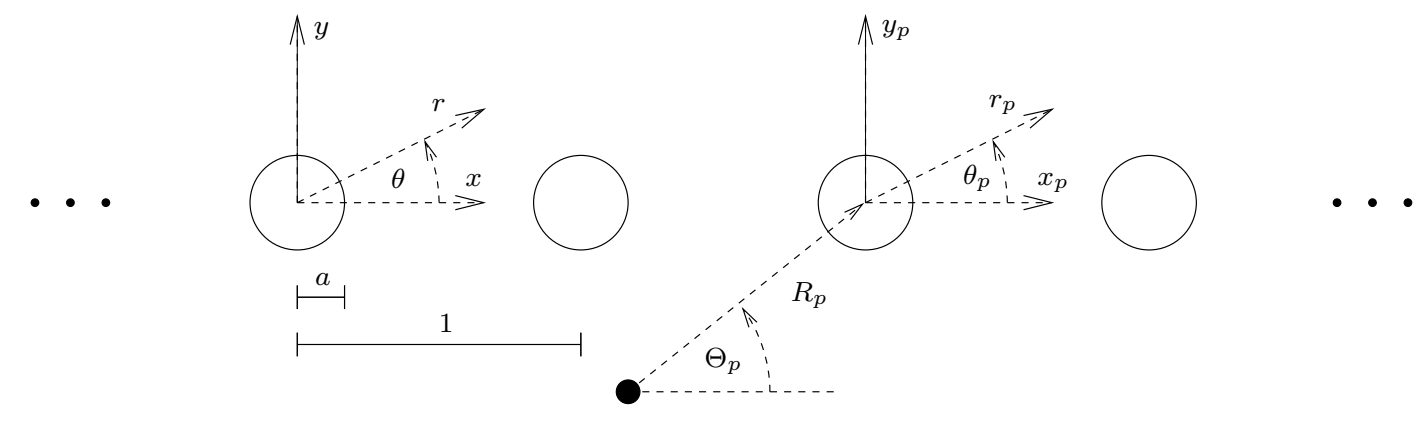

Figure 1: A section of the infinite array. In the $(x, y)$ plane, the scatterers are located at $(p, 0)$ for integer $p$, and the line source $(\bullet)$ at the point $(X, Y)$.

and

$$
\phi^{\mathrm{s}}=\sum_{p=-\infty}^{\infty} \sum_{n=-\infty}^{\infty} L_{n}^{p} \mathrm{H}_{n}\left(k r_{p}\right) \mathrm{e}^{\mathrm{i} n \theta_{p}}
$$

respectively. Here, $S=\sqrt{(x-X)^{2}+(y-Y)^{2}},\left(r_{p}, \theta_{p}\right)$ represents a shifted set of polar co-ordinates with its origin at the centre of scatterer $p$ and $\mathrm{H}_{n}(\cdot)$ represents the $n$th order Hankel function of the first kind. All of the terms in (1) satisfy the Helmholtz equation

$$
\left(\nabla^{2}+k^{2}\right) \phi=0
$$

and on the surface of the scatterers we have the Neumann boundary condition

$$
\partial \phi^{\mathrm{t}} / \partial r_{p}=0, \quad p \in \mathbb{Z}
$$

The linear system satisfied by the coefficients $L_{n}^{p}$ can easily be deduced from Linton \& McIver [10, eqn (6.7)]. Thus, we omit the right-hand side due to plane wave forcing, and include an extra 'scatterer' for which $L_{m}=\delta_{m 0}$, to obtain

$$
L_{m}^{p}+Z_{m} \sum_{\substack{j=-\infty \\ j \neq p}}^{\infty} \sum_{n=-\infty}^{\infty} L_{n}^{j} X_{n-m}^{j p} \mathrm{H}_{n-m}(k|j-p|)=(-1)^{m+1} Z_{m} \mathrm{e}^{-\mathrm{i} m \Theta_{p}} \mathrm{H}_{m}\left(k R_{p}\right), \quad m, p \in \mathbb{Z},
$$

where

$$
X_{n}^{j p}= \begin{cases}(-1)^{n} & : j>p \\ 1 & : j<p .\end{cases}
$$

The constants $Z_{n}$ are given by

$$
Z_{n}=\mathrm{J}_{n}^{\prime}(k a) / \mathrm{H}_{n}^{\prime}(k a),
$$

and the spacing of the cylinders is such that

$$
k<\pi
$$


in this regime equation (6) cannot easily be solved by truncation since the coefficients $L_{n}^{p}$ do not tend to zero as $|p| \rightarrow \infty$ due to the presence of surface waves.

The overall problem can easily be split into components that are symmetric and antisymmetric about $y=0$. To obtain the symmetric system, the factor $\mathrm{e}^{-\mathrm{i} m \Theta_{p}}$ on the right-hand side of (6) is replaced by $\cos \left(m \Theta_{p}\right)$, and the set of unknown coefficients is then reduced via

$$
L_{-m}^{p}=(-1)^{m} L_{m}^{p}
$$

Similarly, the antisymmetric system is obtained by replacing $\mathrm{e}^{-\mathrm{i} m \Theta_{p}}$ with $-\mathrm{i} \sin \left(m \Theta_{p}\right)$ and applying the identity

$$
L_{-m}^{p}=(-1)^{m+1} L_{m}^{p}
$$

Note that $L_{0}^{p}=0$ for all $p$ in this case. If the source is positioned so that $X=0$, horizontal symmetry leads to the further reduction

$$
L_{-m}^{-p}=L_{m}^{p}
$$

The summations appearing in (3) and (6) are of two different types. On the interior, we have an order summation (over $n$ ) and as $|n|$ increases, the coefficients $Z_{n}$, and therefore the unknowns $L_{n}^{p}$, rapidly converge to zero [11, $\left.§ 9.3 .1\right]$. The exterior sum is of spatial type, and converges much more slowly, relying on the increasing size of the argument of the Hankel function. We therefore aim to treat these sums analytically; in doing so we require the integral representation [12]

$$
\mathrm{H}_{n}(k r) \mathrm{e}^{\mathrm{i} n \theta}=\frac{(-\mathrm{i})^{n+1}}{\pi} \int_{-\infty}^{\infty}\left[\frac{\alpha-\gamma(\alpha)}{k}\right]^{n \operatorname{sgn}(y)} \mathrm{e}^{-\gamma(\alpha)|y|+\mathrm{i} \alpha x} \frac{\mathrm{d} \alpha}{\gamma(\alpha)} .
$$

Here, $\gamma(\alpha)=\left(\alpha^{2}-k^{2}\right)^{1 / 2}$, with $\gamma(0)=-\mathrm{i} k$. Note that

$$
\arccos (\alpha / k)=-\mathrm{i} \log [\alpha-\gamma(\alpha)]+\mathrm{i} \log k,
$$

for a suitably defined continuation of the inverse cosine to the cut plane. By writing

$$
k=k_{r}+\mathrm{i} \epsilon,
$$

where $k_{r}$ is real, the singularities are removed from the real line, and taking the limit $k \rightarrow k_{r}$ determines the manner in which the path of integration should be indented. Thus, the branch cuts are positioned along the lines $\alpha= \pm k(1+\mathrm{i} u)$, with $u>0$, and the path of integration is indented so as to pass above $\alpha=-k$ and below $\alpha=k$. Evidently, the integral in (13) converges for all values of $y$ except 0 (for $n=0$ it converges everywhere except $x=y=0$ ). Finally, we introduce the function

$$
\psi_{j}(\alpha)=\arccos \left[\frac{1}{k}(\alpha+2 j \pi)\right], \quad j \in \mathbb{Z},
$$

and when no ambiguity can arise we write $\psi_{j}$ in place of $\psi_{j}(\alpha)$. For real $\alpha, \psi_{j}$ is located on one of the line sections $[0, \pi], \mathrm{i} u$ and $\pi-\mathrm{i} u$, with $u>0$. Note that, for all $j$,

$$
k \sin \psi_{j}=\mathrm{i} \gamma\left(k \cos \psi_{j}\right) .
$$




\section{Rayleigh-Bloch waves}

The infinite array can support trapped modes known as Rayleigh-Bloch waves, which propagate without loss in $x$. The form of these waves can be obtained as follows. First, we remove the right-hand side from equation (6) and write

$$
L_{n}^{p}=\mathrm{e}^{\mathrm{i} p \tilde{\beta}} \tilde{B}_{n}
$$

for $\tilde{\beta} \in \mathbb{R}$. After some manipulation, we obtain

$$
\tilde{B}_{m}+Z_{m} \sum_{n=-\infty}^{\infty} \tilde{B}_{n} \sigma_{n-m}(\tilde{\beta})=0, \quad m \in \mathbb{Z}
$$

with $\tilde{B}_{m} \neq 0$ for at least one $m$, and

$$
\sigma_{n}(\tilde{\beta})=\sum_{j=1}^{\infty}\left[\mathrm{e}^{-\mathrm{i} j \tilde{\beta}}+(-1)^{n} \mathrm{e}^{\mathrm{i} j \tilde{\beta}}\right] \mathrm{H}_{n}(j k) .
$$

This quantity is known as a Schlömilch series; in this form its convergence properties are very poor, however formulae which enable $\sigma_{n}$ to be be computed efficiently are available; see $[13,14]$ and appendix A. RB waves with $|\tilde{\beta}| \leq k$ have not been found, and, furthermore, if $\tilde{\beta}$ gives rise to a right propagating $\mathrm{RB}$ wave, then $-\tilde{\beta}$ yields the same wave carrying energy to the left; this has the coefficient $(-1)^{n} \tilde{B}_{n}$ in place of $\tilde{B}_{n}$. In view of the evident $2 \pi$ periodicity, we may impose the restriction

$$
k<\tilde{\beta}<\pi .
$$

Solutions to (19) satisfying (21) give rise to right propagating RB modes and there may be two of these at most; one that is symmetric about $y=0$ and one that is antisymmetric. Computations show that symmetric modes exist for all cylinder sizes $0<a<0.5$, whereas antisymmetric modes exist only if the cylinders are sufficiently large $(a \gtrsim 0.403)$. For a given value of $a, \mathrm{RB}$ waves only exist for a range of values of $k$; symmetric modes in the range

$$
0<k<k_{\max }^{\mathrm{s}}<\pi
$$

and antisymmetric modes in the range

$$
k_{\min }^{\mathrm{a}}<k<k_{\max }^{\mathrm{a}}<\pi
$$

It turns out that there are three distinct regimes: for $a \lesssim 0.403$ only symmetric modes are possible; for $0.403 \lesssim a \lesssim 0.459$ we have $k_{\max }^{\mathrm{s}}<k_{\min }^{\mathrm{a}}$ and so it is possible to have symmetric and antisymmetric modes, but not for the same value of $k$; finally when $0.459 \lesssim a<0.5$ we have $k_{\max }^{\mathrm{s}}>k_{\min }^{\mathrm{a}}$ and hence it is only in this parameter range that it is possible to excite both symmetric and antisymmetric modes at the same time. Figure 2 shows values of $k_{\max }^{\mathrm{s}}$, $k_{\min }^{\mathrm{a}}$ and $k_{\max }^{\mathrm{a}}$ for varying scatterer radius $a$. Note that as $a \rightarrow 0.5$ the gaps between the cylinders shrink to zero. At $a=0.5$ there is no connection between the regions on each side of the array and the symmetric and antisymmetric modes are essentially the same. To 


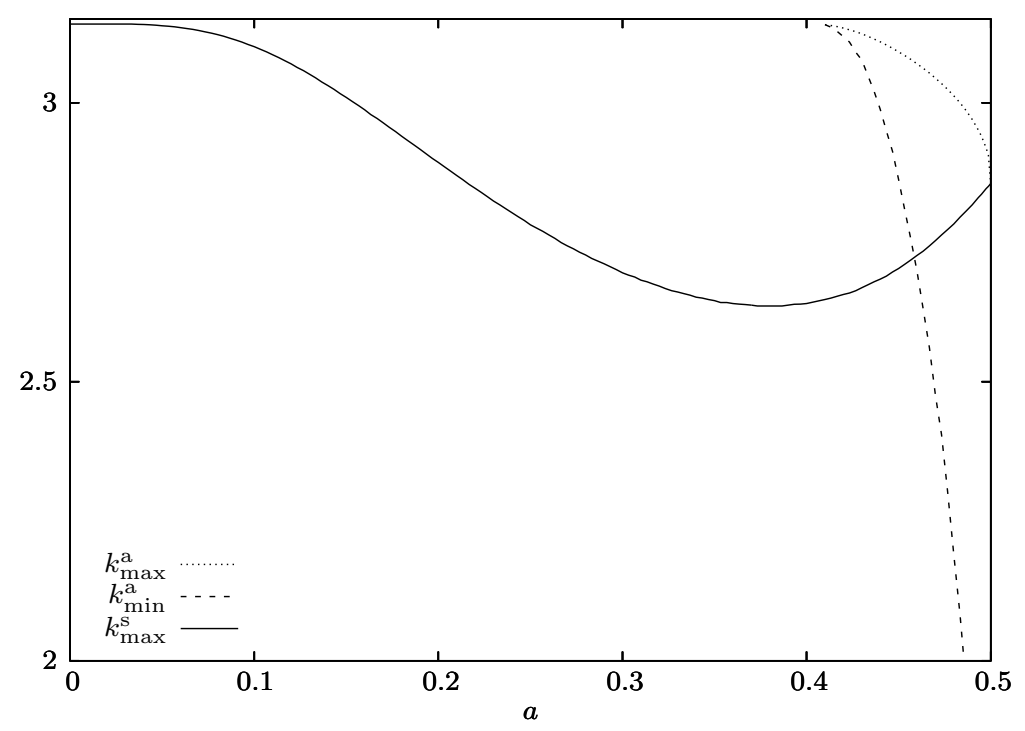

Figure 2: $k_{\min }$ and $k_{\max }$ for symmetric and antisymmetric RB modes.

simplify our subsequent discussion, we will henceforth assume that there is only one type of $\mathrm{RB}$ wave (symmetric or antisymmetric) present, that is there exists a single nontrivial solution to (19) for which $\tilde{\beta}$ satisfies (21). Cases where two modes exist can be dealt with by splitting the problem into parts symmetric and antisymmetric about $y=0$ (c.f. $\S 2$ ).

The problem of actually locating $\tilde{\beta}$ can be simplified using the following procedure, due to Evans \& Porter [15]. It follows from equations (16-17), (21), and (A3-A5) that $\cos \tilde{\psi}_{j}$ is positive real for all $j$, whereas $\sin \tilde{\psi}_{j}$ is positive imaginary. Hence,

$$
\sigma_{n}(\tilde{\beta})=-\delta_{n 0}+\mathrm{i}^{n+1} \lambda_{n}(\tilde{\beta}),
$$

where $\lambda_{n}$ is real. Using this in (19), and multiplying by $\mathrm{i}^{m} /\left(1-Z_{m}\right)$ yields

$$
\left(\mathrm{i}^{m} \tilde{B}_{m}\right)+\frac{\mathrm{J}_{m}^{\prime}(k a)}{\mathrm{Y}_{m}^{\prime}(k a)} \sum_{n=-\infty}^{\infty}\left(\mathrm{i}^{n} \tilde{B}_{n}\right) \lambda_{n-m}(\tilde{\beta})=0, \quad m \in \mathbb{Z} ;
$$

a linear system with real coefficients. Once obtained, the coefficients $\tilde{B}_{n}$ are normalised so that

$$
\sum_{n=-\infty}^{\infty}\left|\tilde{B}_{n}\right|^{2}=1
$$

Substituting (18) into (3) and using the identity (13) along with the Poisson summation formula, we obtain the modal representation for the RB waves, valid for $y>0$, thus

$$
\phi^{\mathrm{rb}}=\Gamma^{ \pm} \sum_{j=-\infty}^{\infty}[ \pm] \tilde{A}_{j} \mathrm{e}^{\mathrm{i} k\left( \pm x \cos \tilde{\psi}_{j}+y \sin \tilde{\psi}_{j}\right)} .
$$

Here, the upper and lower signs refer to the right and left propagating waves, respectively, and the symbol in square brackets appears in the antisymmetric case only. In addition we have introduced the notation

$$
\tilde{\psi}_{j}=\psi_{j}(\tilde{\beta}) .
$$


The quantities $\Gamma^{ \pm}$represent arbitrary (complex) amplitude coefficients, and $\tilde{A}_{j}$ is given by

$$
\tilde{A}_{j}=\frac{2}{k \sin \tilde{\psi}_{j}} \sum_{n=-\infty}^{\infty}(-\mathrm{i})^{n} \tilde{B}_{n} \mathrm{e}^{\mathrm{i} n \tilde{\psi}_{j}} .
$$

Note that this is pure imaginary. Equivalent results for $y<0$ can be deduced by symmetry, but the modal form is not valid on $y=0$. The $\mathrm{RB}$ wave evidently propagates without loss in the $x$ direction, and decays exponentially as $y$ is increased.

The presence of these waves at the extremities of the array accounts for the fact that the coefficients $L_{m}^{p}$ do not tend to zero as $|p| \rightarrow \infty$. After writing

$$
L_{m}^{p}= \begin{cases}\hat{L}_{m}^{p}+\Gamma^{+} \tilde{B}_{m} \mathrm{e}^{\mathrm{i} p \tilde{\beta}} & : p \geq 0, \\ \hat{L}_{m}^{p}+\Gamma^{-}(-1)^{m} \tilde{B}_{m} \mathrm{e}^{-\mathrm{i} p \tilde{\beta}} & : p<0,\end{cases}
$$

a linear system for $\hat{L}_{m}^{p}$ can be formed by substitution into (6). The left-hand side is the same as that for $L_{m}^{p}$, and a correction term $Q_{m}^{p}$ must be added to the right-hand side. It is not difficult to show that

$$
Q_{m}^{p}=(-1)^{m} Z_{m} \sum_{n=-\infty}^{\infty} \tilde{B}_{n}\left[\Gamma^{-} \mathrm{e}^{-\mathrm{i} p \tilde{\beta}} s_{n-m}^{|p|}(-\tilde{\beta})-(-1)^{n} \Gamma^{+} \mathrm{e}^{\mathrm{i} p \tilde{\beta}} s_{n-m}^{|p|}(\tilde{\beta})\right]
$$

for $p<0$, whereas for $p \geq 0$, we have

$$
Q_{m}^{p}=Z_{m} \sum_{n=-\infty}^{\infty} \tilde{B}_{n}\left[\Gamma^{+} \mathrm{e}^{\mathrm{i} p \tilde{\beta}} s_{n-m}^{1+p}(-\tilde{\beta})-(-1)^{n} \Gamma^{-} \mathrm{e}^{-\mathrm{i} p \tilde{\beta}} s_{n-m}^{1+p}(\tilde{\beta})\right],
$$

with

$$
s_{n}^{p}(\alpha)=\sum_{j=p}^{\infty} \mathrm{H}_{n}(k j) \mathrm{e}^{\mathrm{i} j \alpha} .
$$

In both cases, the expression for $Q_{m}^{p}$ has been simplified by using (19). Efficient methods for evaluating the function $s_{n}^{p}(\alpha)$ are given in [16]. The linear system for $\hat{L}_{m}^{p}$ can then be solved by truncation, since the unknowns now decay as $|p| \rightarrow \infty$. Although the solution to this system is not required in the determination of $\Gamma^{ \pm}$, it acts as a useful check on the correctness of the numerical codes, and can be used to compute the field close to the scatterers.

\section{Integral representation of the solution}

We now represent the unknown coefficients $L_{n}^{p}$ as Fourier integrals, thus

$$
L_{n}^{p}=\frac{1}{\mathrm{i} \pi} \int_{-\infty}^{\infty} f_{n}(\alpha ; X ; Y) \mathrm{e}^{\mathrm{i} \alpha p} \mathrm{~d} \alpha,
$$

where the function $f_{n}$ is independent of the scatterer number $p$, and substitute this into the linear system (6). Consider the spatial summation which appears on the left-hand side. A straightforward calculation yields

$$
\sum_{\substack{j=-\infty \\ j \neq p}}^{\infty} L_{n}^{j} X_{n-m}^{j p} \mathrm{H}_{n-m}(k|j-p|)=\frac{1}{\mathrm{i} \pi} \int_{-\infty}^{\infty} f_{n}(\alpha ; X ; Y) \sigma_{n-m}(\alpha) \mathrm{e}^{\mathrm{i} \alpha p} \mathrm{~d} \alpha,
$$


where $\sigma_{n}$ is the Schlömilch series (20). For the right-hand side, we note that

$$
R_{p} \cos \Theta_{p}=p-X \text { and } R_{p} \sin \Theta_{p}=-Y,
$$

therefore replacing $n$ with $-m$ in (13) and applying the identity

$$
\frac{\alpha+\gamma(\alpha)}{k}=\frac{k}{\alpha-\gamma(\alpha)}
$$

shows that

$$
\mathrm{H}_{m}\left(k R_{p}\right) \mathrm{e}^{-\mathrm{i} m \Theta_{p}}=-\frac{\mathrm{i}^{1-m}}{\pi} \int_{-\infty}^{\infty}\left[\frac{\alpha+\gamma(\alpha)}{k}\right]^{m} \mathrm{e}^{-\gamma(\alpha)|Y|-\mathrm{i} \alpha X} \mathrm{e}^{\mathrm{i} \alpha p} \frac{\mathrm{d} \alpha}{\gamma(\alpha)} .
$$

Collecting these results together, we obtain

$$
\begin{aligned}
& \int_{-\infty}^{\infty}\left[f_{m}(\alpha ; X ; Y)+Z_{m} \sum_{n=-\infty}^{\infty} f_{n}(\alpha ; X ; Y) \sigma_{n-m}(\alpha)\right] \mathrm{e}^{\mathrm{i} \alpha p} \mathrm{~d} \alpha \\
&=-Z_{m} \mathrm{i}^{m} \int_{-\infty}^{\infty}\left[\frac{\alpha+\gamma(\alpha)}{k}\right]^{m} \mathrm{e}^{-\gamma(\alpha)|Y|-\mathrm{i} \alpha X} \mathrm{e}^{\mathrm{i} \alpha p} \frac{\mathrm{d} \alpha}{\gamma(\alpha)}
\end{aligned}
$$

and if we write

$$
f_{m}(\alpha ; X ; Y)=\frac{B_{m}(\alpha)}{\gamma(\alpha)} \mathrm{e}^{-\gamma(\alpha)|Y|-\mathrm{i} \alpha X},
$$

then (39) will clearly be solved if

$$
B_{m}(\alpha)+Z_{m} \sum_{n=-\infty}^{\infty} B_{n}(\alpha) \sigma_{n-m}(\alpha)=-Z_{m} \mathrm{i}^{m}\left[\frac{\alpha+\gamma(\alpha)}{k}\right]^{m} .
$$

Hence, the integral representation for the coefficient $L_{n}^{p}$ is

$$
L_{n}^{p}=\frac{1}{\mathrm{i} \pi} \int_{-\infty}^{\infty} \frac{B_{n}(\alpha)}{\gamma(\alpha)} \mathrm{e}^{-\gamma(\alpha)|Y|+\mathrm{i} \alpha(p-X)} \mathrm{d} \alpha ;
$$

this result can also be obtained via the 'array scanning method' [9], though the technique used above is somewhat simpler in this particular case.

Typically, the function $B_{n}(\alpha)$ can be evaluated directly from (41). As noted in $\S 3$, however, there are real values of $\alpha$ for which the matrix on the left-hand side of (41) is singular, i.e. when the homogeneous system (19) possesses a nontrivial solution. At these points, the characteristic polynomial of the matrix has a simple zero, and therefore the system (41) can be solved by allowing the unknown functions $B_{n}(\alpha)$ to possess simple poles. Those whose location is given by $\alpha=\tilde{\beta}+2 j \pi$ and $\alpha=\tilde{\beta}-2 j \pi(j \in \mathbb{Z})$ contribute to the right- and left-propagating $\mathrm{RB}$ waves, respectively.

Consider the pole located at $\alpha=\tilde{\beta}$. By Laurent's theorem, we can write

$$
B_{n}(\alpha)=\frac{b_{n}}{\alpha-\tilde{\beta}}+C_{n}(\alpha),
$$


where $C_{n}$ is regular at the point $\alpha=\tilde{\beta}$. Substituting this into (41), multiplying by $(\alpha-\tilde{\beta})$ and taking the limit $\alpha \rightarrow \tilde{\beta}$ shows that the coefficients $b_{n}$ satisfy the same linear system as $\tilde{B}_{n}$, hence $b_{n}=c^{+} \tilde{B}_{n}$, for some constant $c^{+}$. Equation (41) now becomes

$$
\begin{array}{r}
C_{m}(\alpha)+Z_{m} \sum_{n=-\infty}^{\infty} C_{n}(\alpha) \sigma_{n-m}(\alpha)=\frac{-c^{+}}{\alpha-\tilde{\beta}}\left[\tilde{B}_{m}+Z_{m} \sum_{n=-\infty}^{\infty} \tilde{B}_{n} \sigma_{n-m}(\alpha)\right] \\
-Z_{m} \mathrm{i}^{m}\left[\frac{\alpha+\gamma(\alpha)}{k}\right]^{m}
\end{array}
$$

where all terms on the right-hand side are known, aside from the constant $c^{+}$, and the limit $\alpha \rightarrow \tilde{\beta}$ can be taken using L'Hôpital's rule. (Derivatives of $\sigma_{n}(\alpha)$ can be calculated as shown in appendix A.) In this limit, the matrix of coefficients on the left-hand side becomes singular, and since the functions $C_{n}$ are regular at this point it follows that (44) can be solved if and only if the right-hand side and the (nontrivial) solution to the homogeneous adjoint problem are mutually orthogonal [17, eqns (5.7-5.9)]. That is, if

$$
D_{m}+\sum_{n=-\infty}^{\infty} Z_{n}^{*}(-1)^{n-m} \sigma_{n-m}^{*}(\tilde{\beta}) D_{n}=0, \quad m \in \mathbb{Z}
$$

with $D_{m} \neq 0$ for at least one $m$, then

$$
-\mathrm{i} c^{+} \sum_{m=-\infty}^{\infty} D_{m}^{*} Z_{m}(-\mathrm{i})^{m} \sum_{n=-\infty}^{\infty} \mathrm{i}^{n} \tilde{B}_{n}\left[\frac{\mathrm{d}}{\mathrm{d} \alpha} \lambda_{n-m}(\alpha)\right]_{\alpha=\tilde{\beta}}=\sum_{m=-\infty}^{\infty} D_{m}^{*} Z_{m} \mathrm{i}^{m}\left[\frac{\tilde{\beta}+\gamma(\tilde{\beta})}{k}\right]^{m} .
$$

Here, the superscript ' $*$ ' refers to the complex conjugate, and the function $\lambda_{n}(\alpha)$ is defined as in (24). If we now use (24) in (45) and compare the resulting expression with (25), it follows immediately that

$$
D_{m}=\tilde{B}_{m} / Z_{m}^{*}
$$

Equation (46) therefore yields a unique value for $c^{+}$, which can be obtained by solving a single linear system involving only an order summation. Note that $c^{+}$is pure imaginary, since $\mathrm{i}^{n} \tilde{B}_{n}$ and $\lambda_{n}(\tilde{\beta})$ are real $(\S 3)$. Finally, we observe from equations (41), (37) and (A2) that

$$
B_{-n}(-\alpha)=B_{n}(\alpha) \text {. }
$$

Now, as noted in $\S 3$, the left-propagating wave has the coefficient $(-1)^{n} \tilde{B}_{n}$ in place of $\tilde{B}_{n}$. Thus, if we write

$$
B_{n}(\alpha)=\frac{c^{+} \tilde{B}_{n}}{\alpha-\tilde{\beta}}+\frac{c^{-}(-1)^{n} \tilde{B}_{n}}{\alpha+\tilde{\beta}}+C_{n}(\alpha),
$$

where now $C_{n}(\alpha)$ is regular at $\alpha= \pm \tilde{\beta}$, then, in view of (48), changing $\alpha$ to $-\alpha$ and $n$ to $-n$ yields

$$
c^{+} \tilde{B}_{-n}=c^{-}(-1)^{n+1} \tilde{B}_{n}
$$

and

$$
c^{+} \tilde{B}_{n}=c^{-}(-1)^{n+1} \tilde{B}_{-n} .
$$




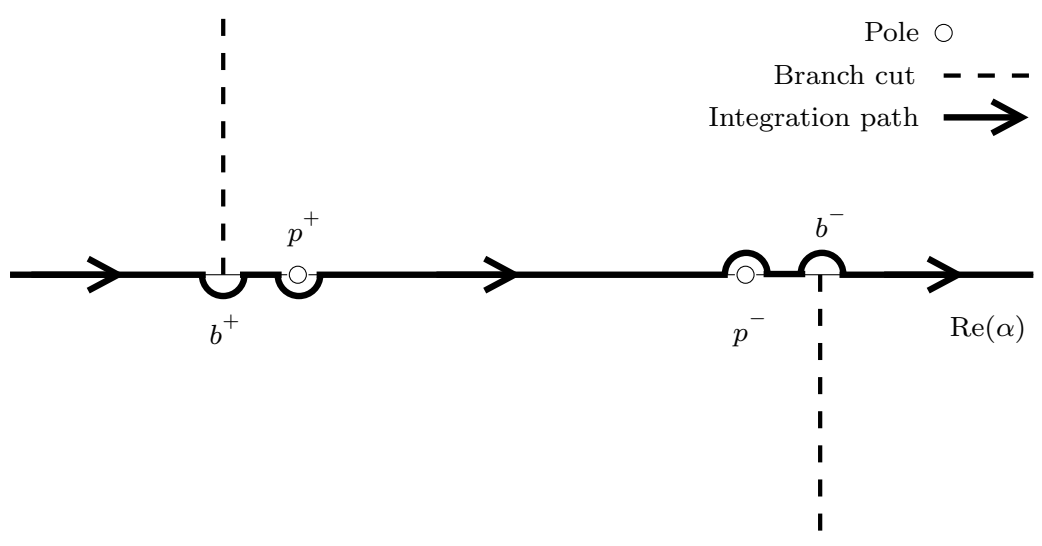

Figure 3: A rectangular strip of the complex plane. The complete plane consists of $2 \pi$ periodic repetitions of this structure. The locations of the singularities are given by $b^{+}=$ $k+2 j \pi, b^{-}=-k+2(j+1) \pi$, and $p^{+}=\tilde{\beta}+2 j \pi, p^{-}=-\tilde{\beta}+2(j+1) \pi$.

Hence $c^{+}= \pm c^{-}$. Equations (10) and (11) now show that the upper and lower signs apply in the antisymmetric and symmetric cases, respectively. Other residues can be obtained by simply substituting $\tilde{\beta}+2 j \pi, j \in \mathbb{Z}$ in place of $\tilde{\beta}$ in the above analysis.

The singularity structure of the function $B_{n}(\alpha)$, and its relationship to the integral (42) may now be determined. In addition to poles $B_{n}(\alpha)$ possesses branch points which it inherits from the function $\sigma_{n}(\alpha)$ via (41). The location of these in the $\alpha$ plane is determined in appendix A; the path of integration passes below the branch points given by (A8) and above those given by (A9). Similarly, the path of integration is indented to pass below those poles whose location is given by $\alpha=\tilde{\beta}+2 j \pi$ and above those given by $\alpha=-\tilde{\beta}+2 j \pi$, so as to yield right- and left-propagating RB modes for $p \gg 0$ and $p \ll 0$ respectively. The strip

$$
k+2 j \pi \leq \operatorname{Re}[\alpha] \leq-k+2(j+1) \pi
$$

is illustrated in figure 3. The complete complex plane consists of periodic repetitions of this region. As we are concerned with the case $k<\pi$ and since $\tilde{\beta} \in(k, \pi)$, the branch points lie outside the interval $(-k, k)$ and do not interfere with the calculation of RB amplitude.

The asymptotic behaviour of (42) for large $|p|$ is easily deduced. Thus, consider the limit $p \rightarrow \infty$. The path of integration is deformed into the upper half plane, and residues are collected from the poles located at $\alpha=\tilde{\beta}+2 j \pi$. The remaining contributions, which make up the coefficient $\hat{L}_{m}^{p}$ in equation (30) come from the branch points $\alpha=k+2 j \pi, j \in \mathbb{Z}$. Results in [18] show that $B_{n}(\alpha)$ remains finite as these points are approached, as does the ratio $B_{n}(\alpha) / \gamma(\alpha)$ as $\alpha \rightarrow \pm k$. All of the branch points are of order one (see appendix A), and the leading contributions from opposing sides of a cut always cancel each other. The same argument applies as $p \rightarrow-\infty$ (in this case the path of integration must be deformed downwards), and it then follows that, as $p \rightarrow \pm \infty$,

$$
\hat{L}_{m}^{p} \sim L_{m}^{ \pm} \frac{\mathrm{e}^{ \pm \mathrm{i} k p}}{p^{3 / 2}} .
$$




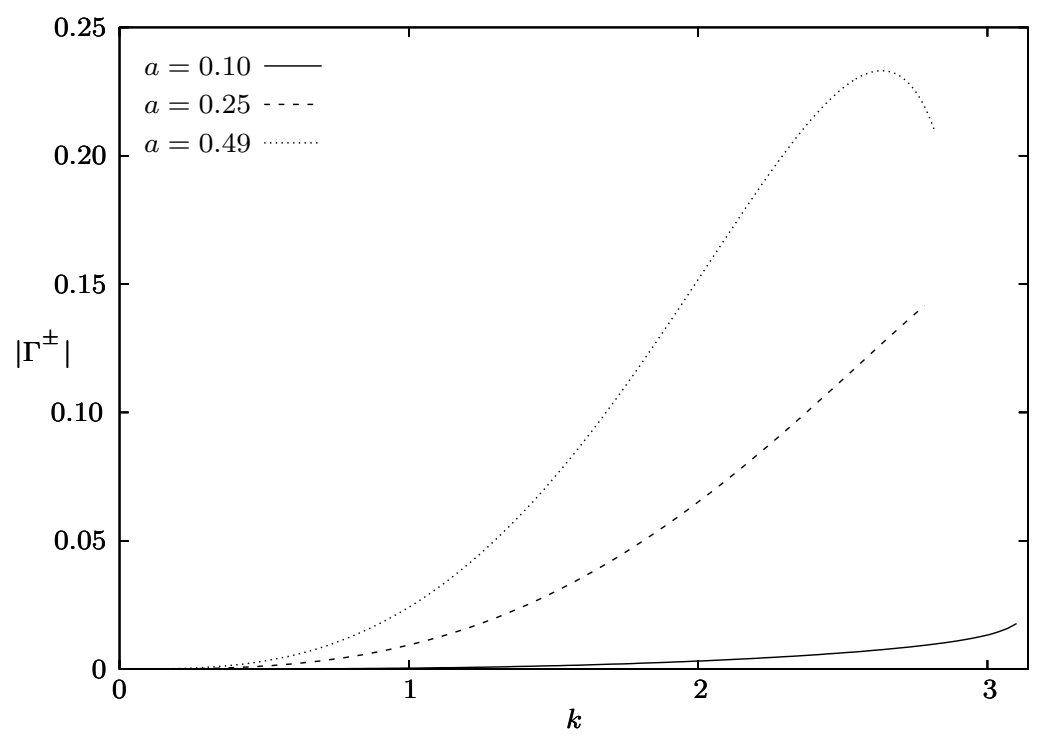

Figure 4: Symmetric Rayleigh-Bloch wave amplitudes for a source positioned at $(0,-1)$.

\section{The far field}

Let us first consider the region where $|x-X|$ is large and $|y| \approx 0$. Deforming the path of integration in (42) into the appropriate half plane, we collect residues to find that

$$
\Gamma^{ \pm}= \pm \frac{2 \mathrm{i}}{k} \sum_{j=-\infty}^{\infty} \frac{c_{j}^{ \pm}}{\sin \tilde{\psi}_{j}} \mathrm{e}^{\mathrm{i} k\left(\sin \tilde{\psi}_{j}|Y| \pm \cos \tilde{\psi}_{j} X\right)} .
$$

Here, $c_{j}^{ \pm}$refers to the constant $c^{ \pm}$in $(46)$, with $\tilde{\beta}$ replaced by $\tilde{\beta}+2 j \pi$ throughout the analysis in $\S 4$. Contributions from the branch points can be neglected, since these decay according to (52). Thus, in this case the dominant contribution to the scattered field is due to the RB waves. The series in (53) converges exponentially, therefore only a small number of terms is required in computing $\Gamma^{ \pm}$to a high degree of accuracy, provided that $Y \not \approx 0$.

Note that $\left|\Gamma^{+}\right|=\left|\Gamma^{-}\right|$so that the amplitudes of the left- and right-propagating RB waves are the same, whatever the source position. Also, if $X=0$, then we have $\Gamma^{+}=\Gamma^{-}$ and $\Gamma^{+}=-\Gamma^{-}$in the (vertically) symmetric and antisymmetric cases, respectively. The amplitudes of the RB waves decay exponentially as $|Y|$, the distance between the source and the array is increased. For moderately large $|Y|$, only the $j=0$ term is required, and in this case the variations in $X$ merely result in a phase shift.

The value of $\left|\Gamma^{ \pm}\right|$for the symmetric RB wave is shown in figure 4 , for three different scatterer sizes, with varying $k$. Here, as in all of our subsequent numerical calculations, the source is positioned at $(X, Y)=(0,-1)$. A corresponding plot for the antisymmetric mode, which exists within a narrower wavenumber spectrum, and only for large scatterers is shown in figure 5. The amplitude tends to increase with frequency, however in the symmetric case with $a=0.49$ it attains its maximum value before the cut-off is reached. Generally, increasing the scatterer size also leads to greater RB wave amplitudes. The final data point on each of the curves in figures 4 and 5 is positioned so that $k_{\max }-k<10^{-8}$ so as to 


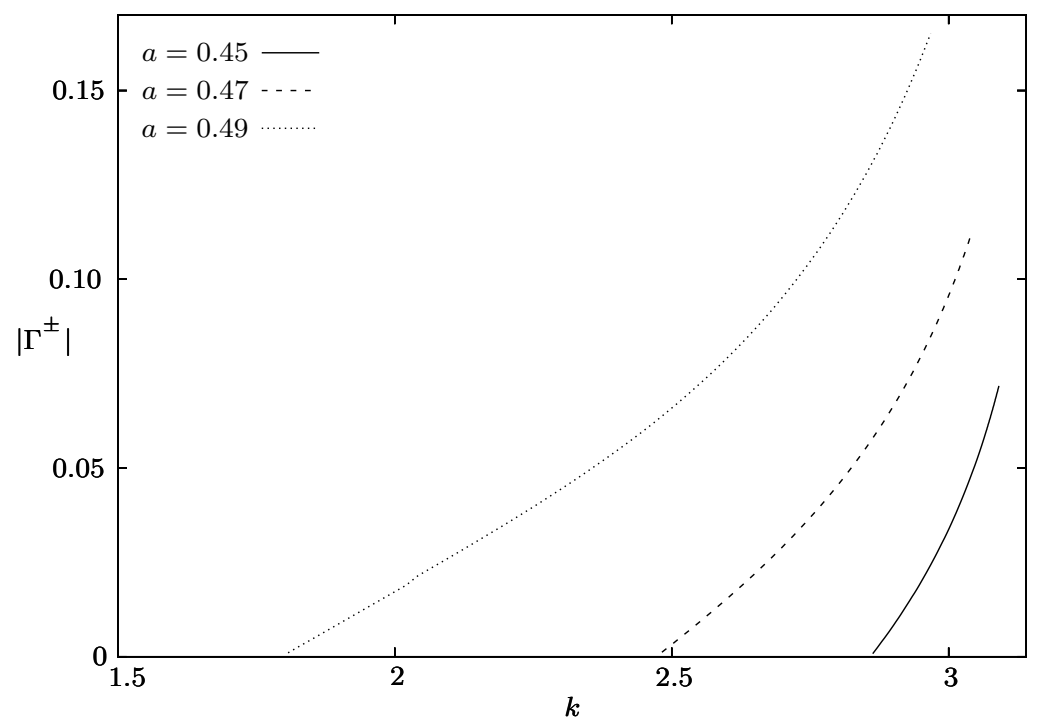

Figure 5: Antisymmetric Rayleigh-Bloch wave amplitudes for a source positioned at $(0,-1)$.

capture any sharp changes in amplitude as the cut-off is approached. No such behaviour was observed; in fact, as $k \rightarrow k_{\max }, \tilde{\beta} \rightarrow \pi$, so that the RB mode tends to a standing wave of finite amplitude. Typically, where both waves are present the symmetric mode has the larger amplitude, as in the case where $a=0.49$. The correctness of these results has been confirmed by truncating (6), and inverting the resulting linear system. Where RB waves are present, the absence of decay in $L_{m}^{p}$ as $p$ is increased can be observed using a relatively small $(\approx 25-30)$ spatial truncation. On adjusting the right-hand side using $(31)$ and $(32)$, the contribution from the RB wave(s) is removed, inducing the unknown coefficients to decay as predicted in (52).

To illustrate the behaviour of $L_{m}^{p}$ as $p \rightarrow \pm \infty$, we define

$$
L_{p}=\sqrt{\sum_{m=-\infty}^{\infty}\left|L_{m}^{p}\right|^{2}},
$$

and likewise for $\hat{L}_{m}^{p}$. Results obtained by inverting (6), truncating the spatial sum at \pm 100 (i.e. using 201 scatterers) are shown in figure 6, with the $y$ axis on a base 10 logarithmic scale. Here, $a=0.49, k=2.5$. Both the symmetric and antisymmetric RB modes are present, therefore the quantity $L_{p}$ does not tend to zero as $p \rightarrow \infty$. In fact, it oscillates slightly about a constant value, which can be seen from figure 4 to be the amplitude of the symmetric wave $(\approx 0.23)$. The plot which we have labeled $\hat{L}_{p}$ is obtained by removing this contribution using (31) and (32). This exhibits qualitatively similar behaviour to $L_{p}$, with the oscillations now centred at the amplitude of the antisymmetric wave, which can be read off from figure $5(\approx 0.06)$. Finally, $\hat{\hat{L}}_{p}$ is calculated by removing the contributions from both of the RB waves; the decay in this quantity with increasing $p$ is evident.

The leading order behaviour of the far field in other regions can be obtained by using 


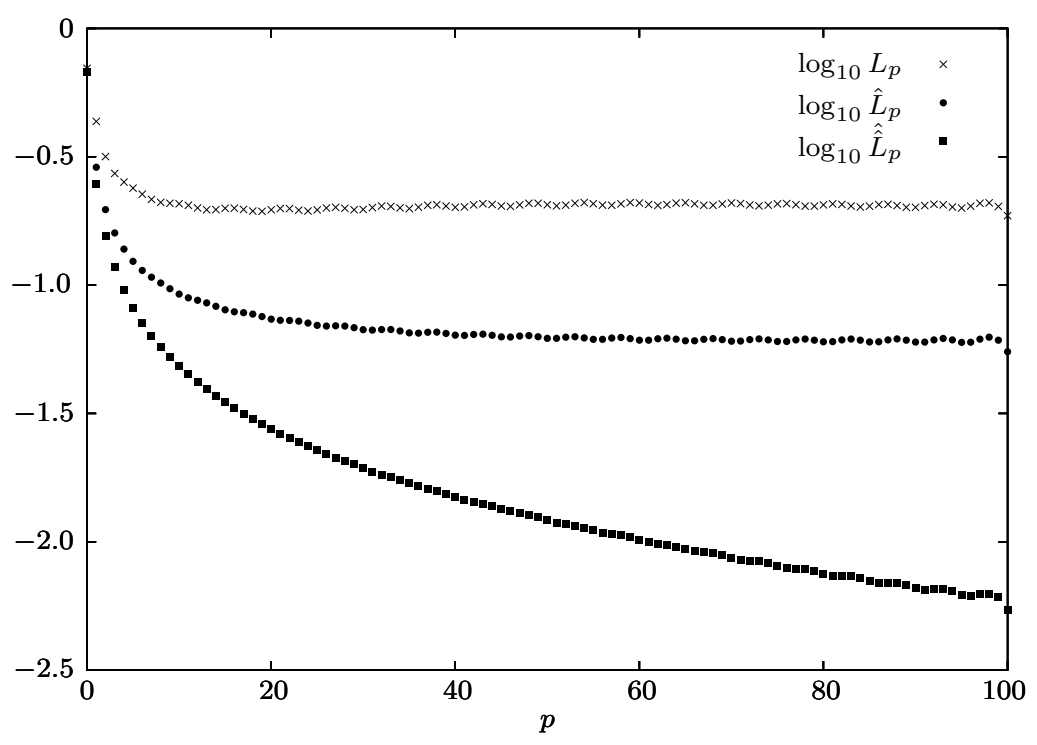

Figure 6: Logarithmic plot illustrating the behaviour of $L_{p}$ as $p$ is increased, with $a=0.49$, $k=2.5$ and the source positioned at $(0,-1)$.

equations (13) and (42) in (3), thus

$$
\begin{aligned}
\phi^{\mathrm{s}}=-\frac{1}{\pi^{2}} \sum_{p=-\infty}^{\infty} \int_{-\infty}^{\infty} \int_{-\infty}^{\infty} \sum_{n=-\infty}^{\infty}(-\mathrm{i})^{n} B_{n}(\alpha) & {\left[\frac{\xi-\gamma(\xi)}{k}\right]^{n \operatorname{sgn}(y)} } \\
& \times \mathrm{e}^{-\gamma(\alpha)|Y|-\mathrm{i} \alpha X} \mathrm{e}^{-\gamma(\xi)|y|+\mathrm{i} \xi x} \mathrm{e}^{\mathrm{i} p(\alpha-\xi)} \frac{\mathrm{d} \alpha \mathrm{d} \xi}{\gamma(\alpha) \gamma(\xi)}
\end{aligned}
$$

The integral over $\xi$ can then be evaluated using the Poisson summation formula, giving

$$
\phi^{\mathrm{s}}=-\frac{\mathrm{i}}{\pi} \sum_{j=-\infty}^{\infty} \int_{-\infty}^{\infty} A_{j}(\alpha) \mathrm{e}^{-\gamma(\alpha)|Y|-\mathrm{i} \alpha X} \mathrm{e}^{-\gamma(\alpha+2 j \pi)|y|+\mathrm{i}(\alpha+2 j \pi) x} \frac{\mathrm{d} \alpha}{\gamma(\alpha)},
$$

in which

$$
A_{j}(\alpha)=\frac{2}{k \sin \psi_{j}(\alpha)} \sum_{n=-\infty}^{\infty}(-\mathrm{i})^{n} B_{n}(\alpha) \mathrm{e}^{\mathrm{i} n \operatorname{sgn}(y) \psi_{j}(\alpha)}
$$

(c.f. equation (29)). The remaining integral may be approximated using the method of steepest descents. First, we show that the branch points do not contribute to the leading order term. The argument is similar to that used in obtaining (52). Thus, the function $A_{j}(\alpha)$ remains bounded at the branch points of the function $B_{n}(\alpha)$ (i.e. in the limit $\sin \psi_{j} \rightarrow 0$ ); the means of demonstrating this can be found in [18]. Also, for $j=0$ the steepest descent path always has the correct orientation with respect to the branch points $\alpha= \pm k$ regardless of whether the source or observer is to be moved far from the origin. When $j \neq 0, A_{j}(\alpha) \rightarrow 0$ as $\alpha \rightarrow \pm k$, and the ratio $A_{j}(\alpha) / \gamma(\alpha)$ is finite, again details are in [18]. The branch points are of order one (see appendix A), and the dominant contributions from opposing sides of a cut always cancel each other. It now follows that, if a descent path should require a diversion around one or more branch points, then the resulting contributions will be one 
asymptotic order smaller than those from the saddle points $(-3 / 2$ as opposed to $-1 / 2)$, and may therefore be neglected. We may also ignore the effect of the poles, since their residues decay exponentially as $|y|$ or $|Y|$ is increased.

In view of this, we need only consider the saddles, deducing their contributions by comparison to (13) with $n=0$, and using the result that, as $k r \rightarrow \infty$,

$$
\mathrm{H}_{0}(k r) \sim F(r)=\sqrt{\frac{2}{\pi k r}} \mathrm{e}^{-\mathrm{i} \frac{\pi}{4}} \mathrm{e}^{\mathrm{i} k r} .
$$

Thus, with $R_{0}$ and $\Theta_{0}$ defined as in (36), we find for $R_{0} \gg 0$ that

$$
\phi^{\mathrm{s}} \sim F\left(R_{0}\right) \sum_{j=-\infty}^{\infty} A_{j}\left(k \cos \Theta_{0}\right) \mathrm{e}^{\mathrm{i} k r \cos \left(\theta-\operatorname{sgn}(y) \psi_{j}\right)},
$$

where the function $\psi_{j}$ is to be evaluated at the point $\alpha=k \cos \Theta_{0}$. It is not difficult to show that, as $R_{0} \rightarrow \infty$,

$$
\phi^{\mathrm{i}} \sim F\left(R_{0}\right) \mathrm{e}^{\mathrm{i} k r \cos \left(\theta-\Theta_{0}\right)}
$$

and this limit provides a useful check on the correctness of (56), since (59) is identical to the exact result for excitation by a plane wave incident at angle $\Theta_{0}$, aside from the presence of the amplitude factor $F\left(R_{0}\right)$.

In calculating the leading order behaviour for $r \gg 0$, some care must be taken so as to obtain results that are valid on both sides of the array. In particular, note that $\psi_{j}(k \cos \theta)$ is an even function of $\theta$, so that if we take $\theta \in[-\pi, \pi]$, then $\psi_{0}(k \cos \theta)=|\theta|$. With this in mind, a straightforward calculation shows that

$$
\phi^{\mathrm{t}} \sim F(r) D(\theta)
$$

where the diffraction coefficient $D(\theta)$ is given by

$$
D(\theta)=\mathrm{e}^{\mathrm{i} k R_{0} \cos \left(\Theta_{0}-\theta\right)}+\sum_{j=-\infty}^{\infty} \mathrm{e}^{\mathrm{i} k R_{0} \cos \left(\Theta_{0}-\psi_{j}\right)} S_{j}(\theta),
$$

in which

$$
S_{j}(\theta)=\frac{\sin \psi_{0}}{\sin \psi_{j}} A_{-j}\left(k \cos \psi_{j}\right) .
$$

Here, the function $\psi_{j}$ is to be evaluated at the point $\alpha=k \cos \theta$. The expression for $S_{j}(\theta)$ can be simplified using (57) to yield

$$
S_{j}(\theta)=\frac{2}{k \sin \psi_{j}} \sum_{n=-\infty}^{\infty}(-\mathrm{i})^{n} B_{n}(k \cos \theta+2 j \pi) \mathrm{e}^{\mathrm{i} n \theta} .
$$

If the coefficients $B_{n}$ are (vertically) symmetric, then we can make the transformations $\theta \rightarrow-\theta$ and $n \rightarrow-n$ to show that $S_{j}(\theta)=S_{j}(-\theta)$, in view of (10). Similar arguments apply in the antisymmetric case, with $S_{j}(\theta)=-S_{j}(-\theta)$. All of the terms in the series in (62) except the $j=0$ term vanish in the limit $\sin \theta \rightarrow 0$ and decay exponentially as $\sin \theta$ is increased. On the dark side of the array, the quantity $1+S_{0}(\theta)$ is the amplitude of a single 


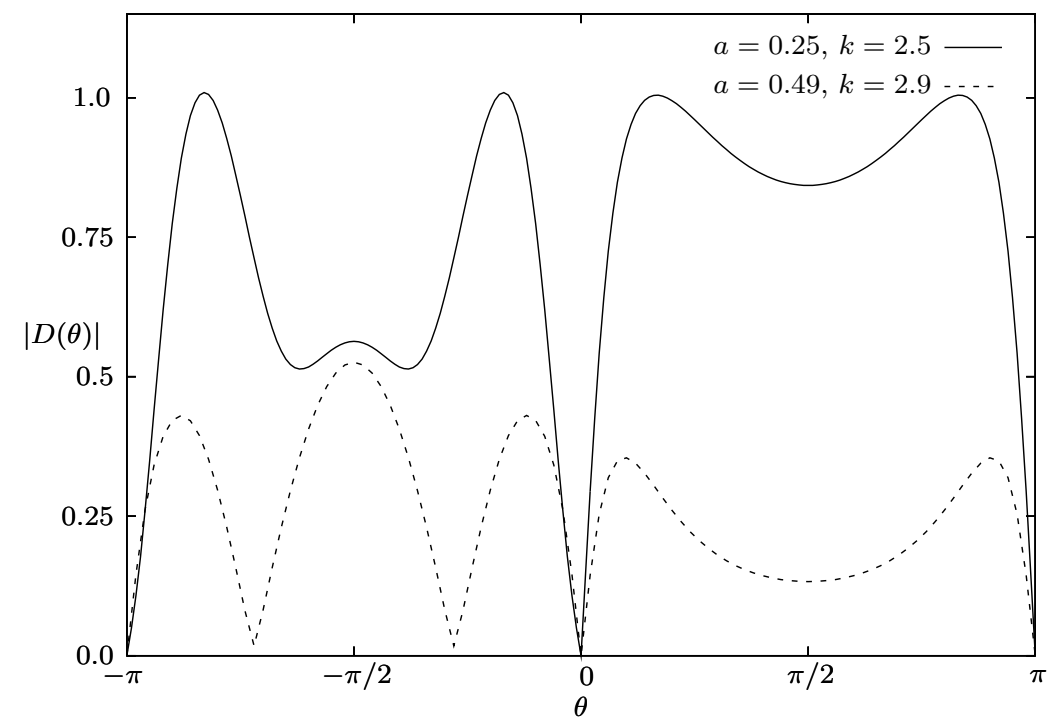

Figure 7: Modulus of the diffraction coefficient $D(\theta)$. The source is positioned at $(X . Y)=$ $(0,-1)$.

circular wave emanating from the source point; this can be thought of as the transmitted field. For $y<0$, i.e. on the lit side, the incident field is present, and there is also a reflected field emanating from $(X,-Y)$, with amplitude $S_{0}(\theta)$. Results in [18] show that

$$
\lim _{\alpha \rightarrow \pm k} A_{0}(\alpha)=-1
$$

meaning that the leading order contributions from the saddle points and the incident field cancel each other at the extremities of the array. This is an acoustic analogue of the Lloyd's mirror effect in optics; see [19, p.519].

The modulus of the diffraction coefficient is shown in figure 7 , for two different sets of parameters. In the first instance, where $a=0.25$ and $k=2.5,|D(\theta)| \approx 1$ except in the vicinity of the array where the circular waves vanish as discussed above, and directly beneath the source, where there is interference between the incident and reflected waves. For $a=0.49$ and $k=2.9$, the effect of transmission is much weaker, as we should intuitively expect. There is also a great deal more interference from the reflected field on the lit side of the array. In particular there are two directions in which $|D(\theta)| \approx 0$; these occur $\theta \approx-\pi / 4$ and $\theta \approx-3 \pi / 4$. In the vicinity of the array, the gradient of $D(\theta)$ is sharper on the dark side of the array than it is on the lit side, hence the circular waves are more significant on the dark side for small values of $|\theta|$.

Figures 8 and 9 show contour plots of the field due to a line source at $(0,-1)$ exciting a large array (201 scatterers). In the first case, we have $a=0.25$ and $k=2.5$ and the formation of a symmetric RB mode is clearly evident. This wave is also shown in isolation, with its complex amplitude coefficient $\Gamma^{+}$computed by assuming the array to be infinite. Both plots are shown on the same amplitude scale, and there is good agreement in both the amplitude and phase of the RB mode. Similarly, in figure 9, the scatterer radius $a$ is 0.49 and the wavenumber $k$ is 2.9 , so that an antisymmetric RB mode is present. As before the 


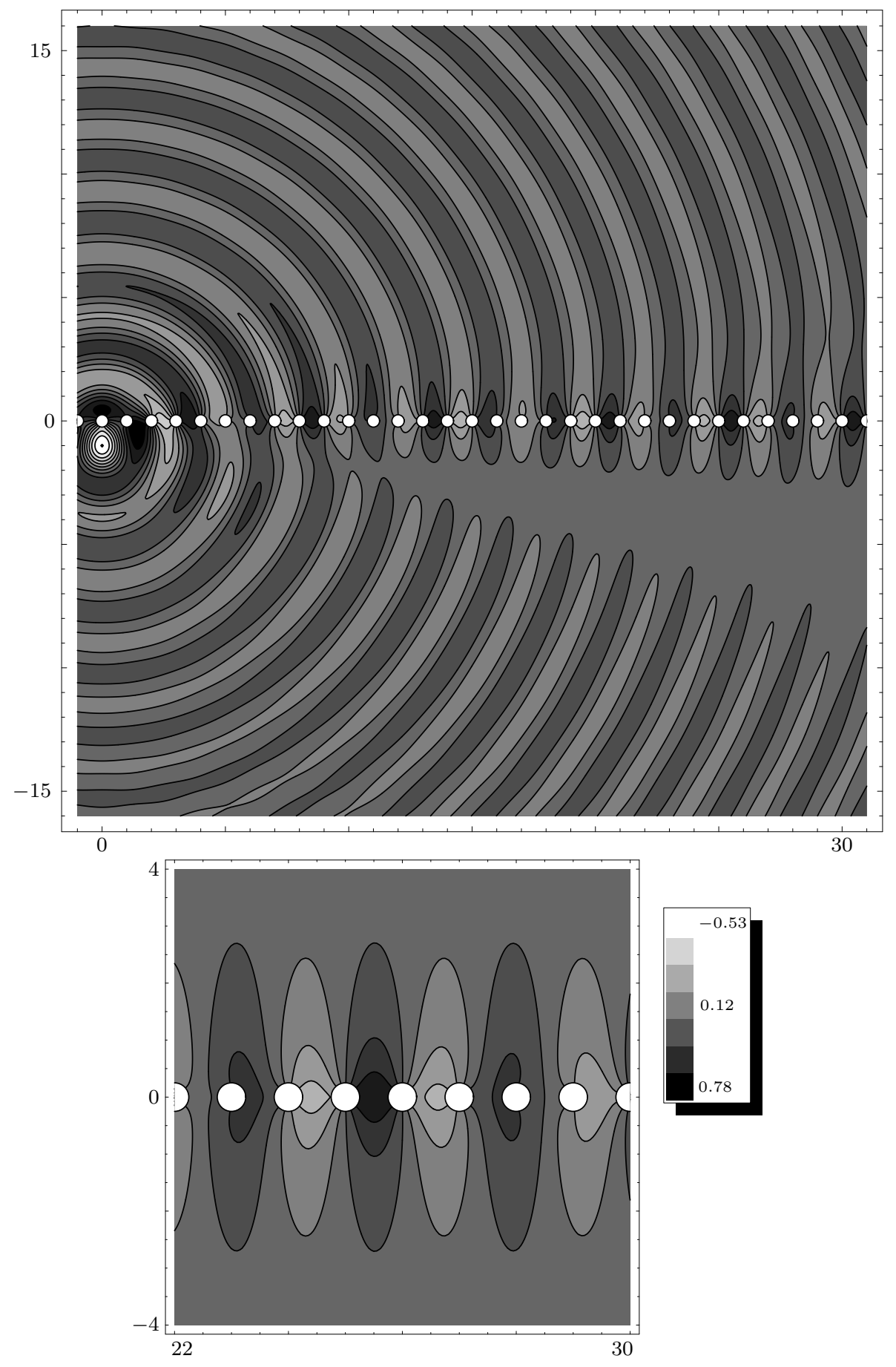

Figure 8: Above: contour plot of $\operatorname{Re}\left[\phi^{\mathrm{t}}\right]$, with $a=0.25$ and $k=2.5$. Note the formation of a symmetric RB wave occurring to the right of the source. Below: A section of the RB wave in isolation (i.e. with no other contributions to the field). The two plots are shown on the same amplitude scale. 


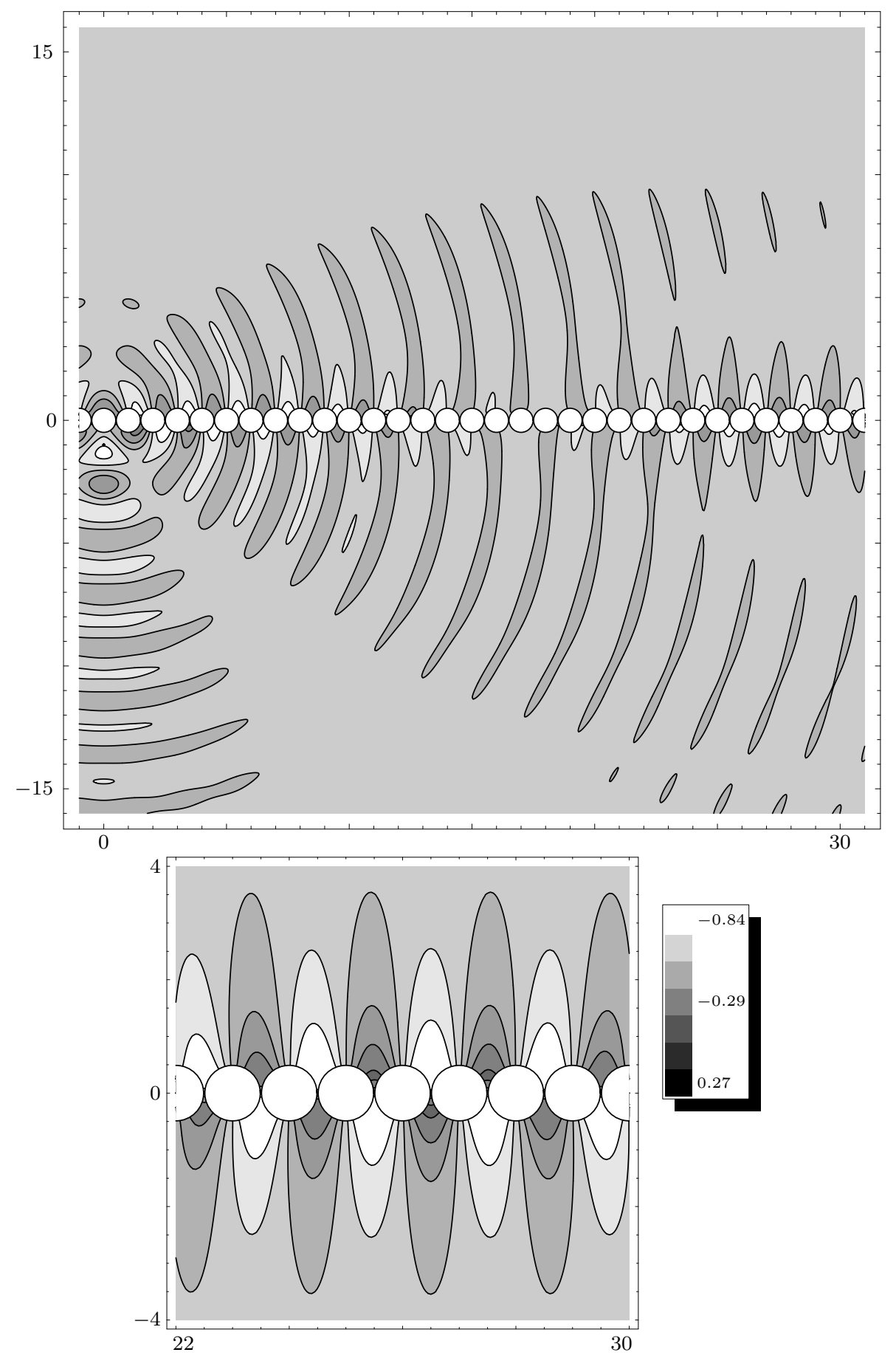

Figure 9: Above: contour plot of $\operatorname{Re}\left[\phi^{\mathrm{t}}\right]$, with $a=0.49$ and $k=2.9$. Note the formation of an antisymmetric RB wave occurring to the right of the source. Below: A section of the RB wave in isolation (i.e. with no other contributions to the field). The two plots are shown on the same amplitude scale. 


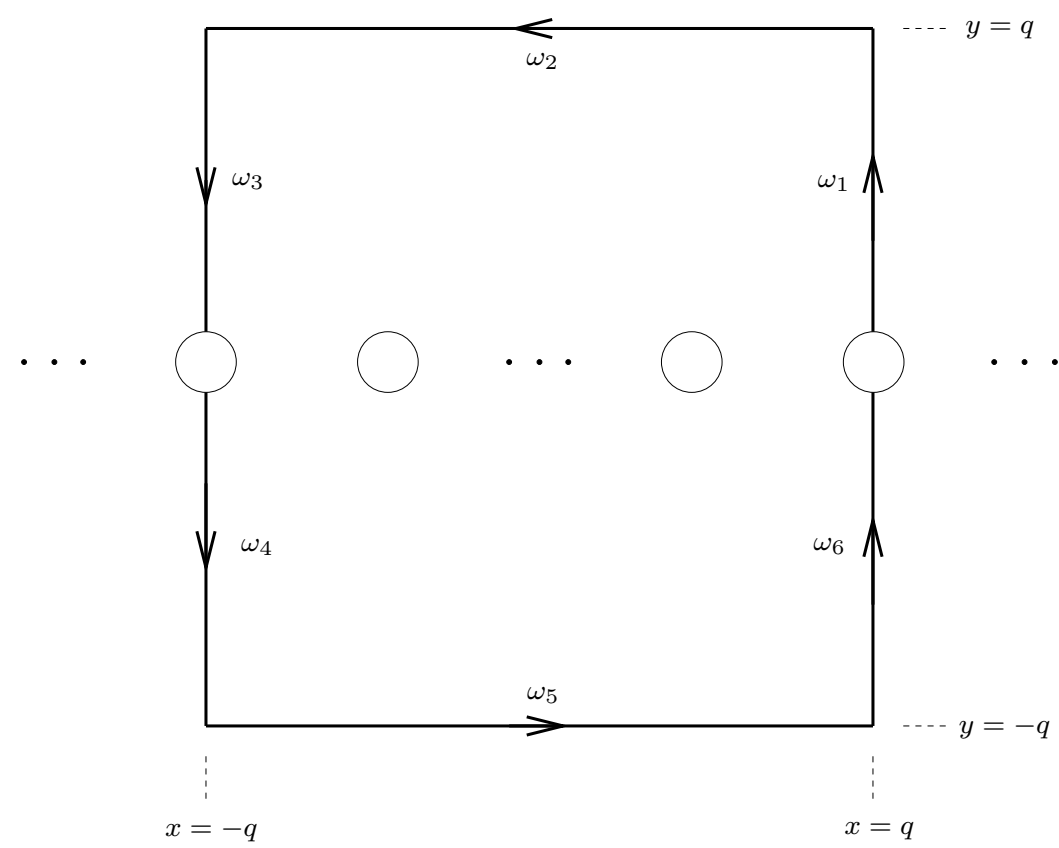

Figure 10: The contour of integration $\Omega$, which is made up from $\omega_{1-6}$.

value of $\Gamma^{+}$appropriate to the infinite array has been used to plot the RB mode in isolation, and again the agreement between the two plots is good. The behaviour of the circular waves is also in agreement with the results for the infinite array.

\section{Energy distribution}

The proportion of energy radiated to infinity by the $\mathrm{RB}$ waves and the other contributions to the field can be determined by applying Green's second identity to $\phi^{\mathrm{t}}$ its complex conjugate in the region bounded by the contour $\Omega$ shown in figure 10, and then taking the limit $q \rightarrow \infty$. Note that the energy radiated by the unit line source is affected by the presence of the array, therefore the sum of these contributions can vary. Thus, the amount of energy radiated by the total field is given by

$$
E^{\mathrm{t}}=C \lim _{q \rightarrow \infty} \int_{\Omega}-\operatorname{Im}\left[\phi^{\mathrm{t}}\left(\frac{\partial \phi^{\mathrm{t}}}{\partial n}\right)^{*}\right] \mathrm{d} s
$$

where $\mathrm{d} n$ is in the direction of the outgoing normal, and $C$ is a positive constant of proportionality which depends upon the particular physical context. Note that there is no contribution from the surfaces of scatterers $\pm q$, since the integrand vanishes here. Also, contributions from (vertically) symmetric and antisymmetric field components are clearly independent of eachother, since (66) evaluates to zero if the integrand is antisymmetric.

Now, let $\phi^{\mathrm{rb}}$ represent the contribution to the total field due to RB waves, and define

$$
\phi^{\mathrm{d}}=\phi^{\mathrm{t}}-\phi^{\mathrm{rb}}
$$


so that $\phi^{\mathrm{d}} \rightarrow 0$ as the distance between source and observer is increased. Using these in (66), we find that the two contributions separate, leaving

$$
E^{\mathrm{t}}=E^{\mathrm{rb}}+E^{\mathrm{d}}
$$

where

$$
E^{\mathrm{rb}}=C \lim _{q \rightarrow \infty} \int_{\Omega}-\operatorname{Im}\left[\phi^{\mathrm{rb}}\left(\frac{\partial \phi^{\mathrm{rb}}}{\partial n}\right)^{*}\right] \mathrm{d} s
$$

and

$$
E^{\mathrm{d}}=C \lim _{q \rightarrow \infty} \int_{\Omega}-\operatorname{Im}\left[\phi^{\mathrm{d}}\left(\frac{\partial \phi^{\mathrm{d}}}{\partial n}\right)^{*}\right] \mathrm{d} s
$$

To show that the cross terms make no contribution, one must first observe that any integral along $\omega_{2}$ and $\omega_{5}$ involving $\phi^{\mathrm{rb}}$ disappears in the limit $q \rightarrow \infty$, due to the exponential decay as $|y|$ is increased, On the remaining sections of $\Omega$, we apply an argument similar to Jordan's lemma. For example

$$
\lim _{q \rightarrow \infty} \int_{\omega_{1}} \phi^{\mathrm{rb}}\left(\frac{\partial \phi^{\mathrm{d}}}{\partial n}\right)^{*} \mathrm{~d} s \leq \lim _{q \rightarrow \infty}\left(\max _{(x, y) \in \omega_{1}}\left|\frac{\partial \phi^{\mathrm{d}}}{\partial x}\right|\right) \int_{a}^{\infty}\left|\phi^{\mathrm{rb}}\right| \mathrm{d} y=0
$$

since the last integral is finite, and $\phi^{\mathrm{d}} \rightarrow 0$ as $x \rightarrow \infty$. The symmetry of the integrand in (69) about $y=0$ now shows that

$$
E^{\mathrm{rb}}=-2 C \int_{a}^{\infty} \operatorname{Im}\left[\phi^{\mathrm{rb}}\left(\frac{\partial \phi^{\mathrm{rb}}}{\partial x}\right)^{*}\right]_{x=q} \mathrm{~d} y+2 C \int_{a}^{\infty} \operatorname{Im}\left[\phi^{\mathrm{rb}}\left(\frac{\partial \phi^{\mathrm{rb}}}{\partial x}\right)^{*}\right]_{x=-q} \mathrm{~d} y,
$$

and an explicit formula is then obtained by using the modal form of the RB wave (27) in the above. Recall that the left- and right-propagating modes are present for $x \ll 0$ and $x \gg 0$, respectively. Since $q \in \mathbb{Z}$, equation (16) shows that

$$
\mathrm{e}^{\mathrm{i} k q \cos \tilde{\psi}_{j}} \mathrm{e}^{-\mathrm{i} k q \cos \tilde{\psi}_{p}}=1
$$

for all $j, p \in \mathbb{Z}$, and hence

$$
E^{\mathrm{rb}}=-4 C\left|\Gamma^{+}\right|^{2} \sum_{j=-\infty}^{\infty} \sum_{p=-\infty}^{\infty} \frac{\cos \tilde{\psi}_{p} \tilde{A}_{j} \tilde{A}_{p}}{\left|\sin \tilde{\psi}_{j}+\sin \tilde{\psi}_{p}\right|} \mathrm{e}^{\mathrm{i} k a\left(\sin \tilde{\psi}_{j}+\sin \tilde{\psi}_{p}\right)} .
$$

This double series is exponentially convergent and therefore its evaluation presents no difficulty.

Equation (70) can be reduced to a real line integral by deforming the contour $\Omega$ into a circle of radius $q$. A simple calculation then shows that

$$
E^{\mathrm{d}}=\frac{2 C}{\pi} \int_{-\pi}^{\pi}|D(\theta)|^{2} \mathrm{~d} \theta
$$

in which $D(\theta)$ is the diffraction coefficient given by (62). This last integral must be evaluated via quadrature. Computed values of $E^{\mathrm{rb}} / E^{\mathrm{t}}$ for the symmetric and anti-symmetric modes 


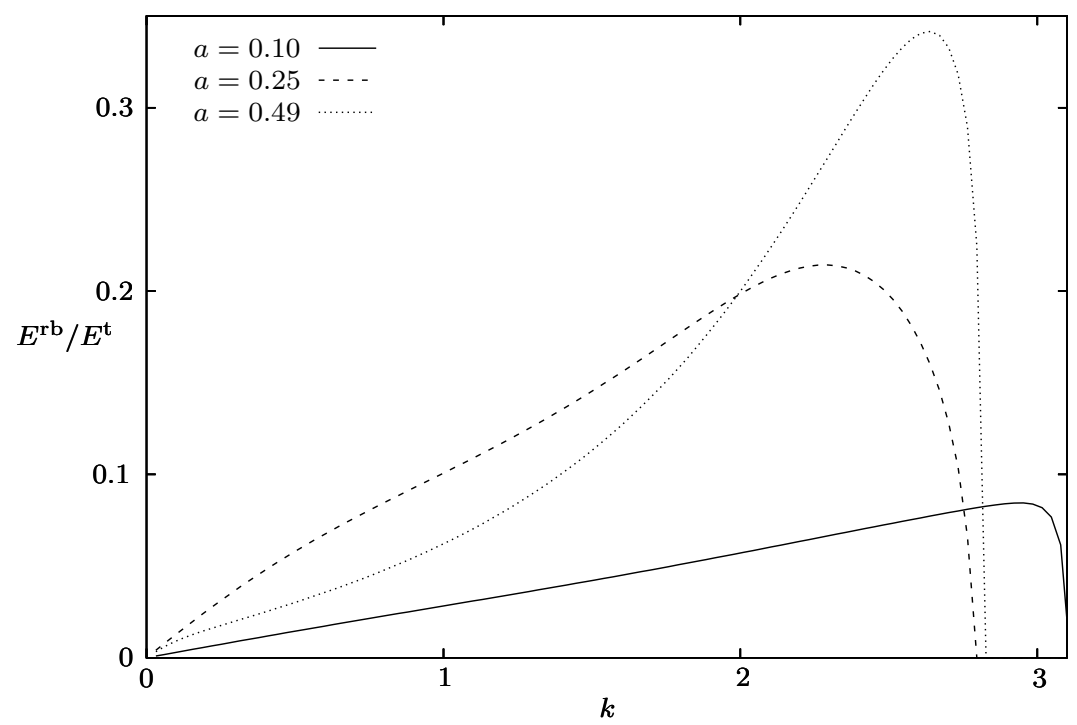

Figure 11: Proportion of incident energy radiated to infinity by the symmetric RB waves. The source is positioned at $(X, Y)=(0,-1)$.

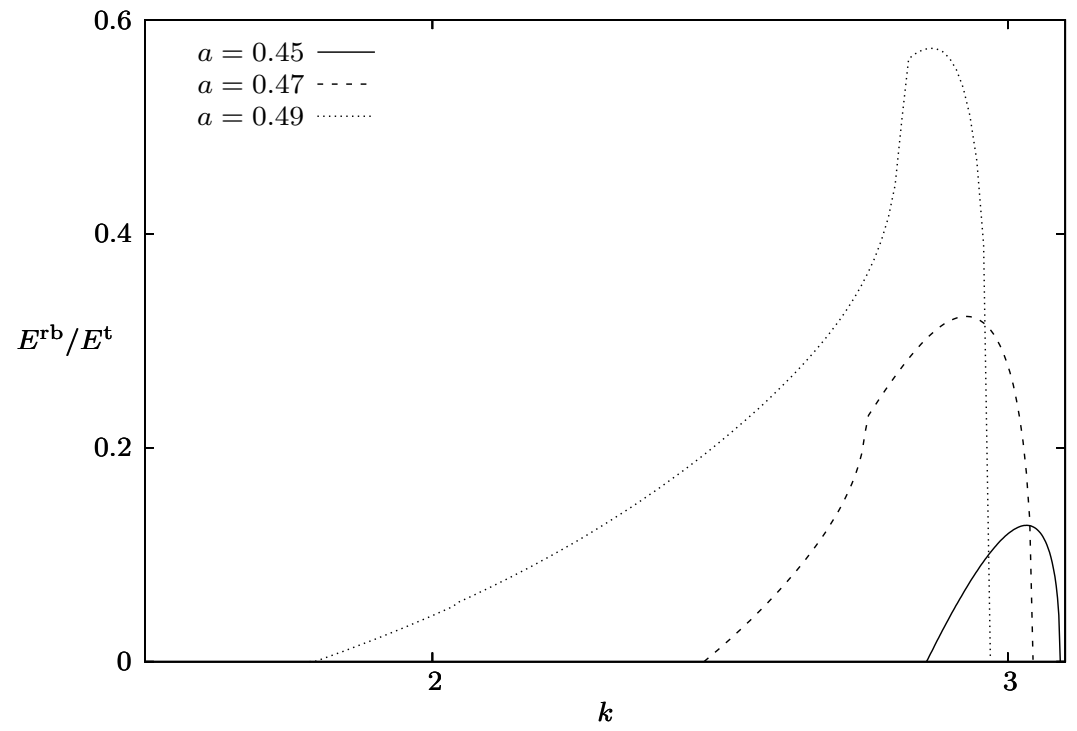

Figure 12: Proportion of incident energy radiated to infinity by the antisymmetric RB waves. The source is positioned at $(X, Y)=(0,-1)$. 
are shown in figures 11 and 12, respectively, for varying wavenumber, and three different scatterer sizes. As $k$ is increased, the proportion of radiated energy carried by the RB waves grows to a maximum and then drops sharply to zero as the cut-off is approached. At the cutoff, $E^{\mathrm{rb}}=0$ since the RB modes are then reduced to standing waves. In the case $a=0.49$, the antisymmetric mode accounts for a particularly large proportion of the radiated energy when $k \approx 2.9$. This is again in agreement with the result for the large finite array shown in figure 9 , where the effect of the circular waves is relatively small.

\section{Conclusions}

The computation of the scattered field that results when an infinite periodic array of closelyspaced rigid circular cylinders is excited by an acoustic line source presents significant computational challenges due to the fact that surface waves are excited along the array. The solution is not periodic and sums over all the cylinders in the array are inevitable in the solution process. Typically these converge very slowly.

In this paper we have shown how the amplitudes of the surface waves that are excited can be computed accurately and efficiently without having to solve the full scattering problem. Interestingly, the amplitudes of the left- and right-propagating surface waves are the same, whatever the source position. The directional dependence of the overall scattered field has also been deduced. By subtracting off the effect of the surface waves with their computed amplitude we can reduce the overall problem to an infinite system of equations in which the coefficients decay like $|p|^{-3 / 2}, p$ being the number of the cylinder in the array. Of particular interest is the proportion of the incident energy that propagates to infinity along the array and we have shown that this can be significant. For cylinders which are almost touching it can be more than $50 \%$.

\section{Acknowledgements}

IT is supported by EPSRC under grant EP/C510941/1. The authors would like to thank Dr M. McIver for useful discussions.

\section{A Schlömilch series and their derivatives}

The Schlömilch series

$$
\sigma_{n}(\alpha)=\sum_{j=1}^{\infty}\left[\mathrm{e}^{-\mathrm{i} j \alpha}+(-1)^{n} \mathrm{e}^{\mathrm{i} j \alpha}\right] \mathrm{H}_{n}(j k)
$$

can be expressed in a form amenable to numerical evaluation using methods found in $[13,14]$. Note that

$$
(-1)^{n} \sigma_{n}(-\alpha)=(-1)^{n} \sigma_{-n}(\alpha)=\sigma_{n}(\alpha)
$$


Thus, with $\psi_{j}$ defined as in (16), for $n=0$, we have

$$
\begin{aligned}
\sigma_{0}(\alpha)=-1-\frac{2 \mathrm{i}}{\pi}\left(C+\ln \frac{k}{4 \pi}\right) & +\frac{2}{k \sin \psi_{0}} \\
& +\sum_{j=1}^{\infty}\left(\frac{2}{k \sin \psi_{j}}+\frac{2}{k \sin \psi_{-j}}+\frac{2 \mathrm{i}}{\pi|j|}\right),
\end{aligned}
$$

where $C$ is Euler's constant. For $n>0$,

$$
\begin{aligned}
\sigma_{2 n}(\alpha)=2(-1)^{n} & \left(\frac{\mathrm{e}^{2 \mathrm{i} n \psi_{0}}}{k \sin \psi_{0}}+\sum_{j=1}^{\infty}\left[\frac{\mathrm{e}^{2 \mathrm{i} n \psi_{j}}}{k \sin \psi_{j}}+\frac{\mathrm{e}^{-2 \mathrm{i} n \psi_{-j}}}{k \sin \psi_{-j}}\right]\right) \\
+ & \frac{\mathrm{i}}{\pi} \sum_{j=0}^{n} \frac{(-1)^{j} 2^{2 j}(n+j-1) !}{(2 j) !(n-j) !}\left(\frac{2 \pi}{k}\right)^{2 j} \mathrm{~B}_{2 j}\left(\frac{\alpha}{2 \pi}\right),
\end{aligned}
$$

and

$$
\begin{array}{r}
\sigma_{2 n-1}(\alpha)=2 \mathrm{i}(-1)^{n}\left(\frac{\mathrm{e}^{\mathrm{i}(2 n-1) \psi_{0}}}{k \sin \psi_{0}}+\sum_{j=1}^{\infty}\left[\frac{\mathrm{e}^{\mathrm{i}(2 n-1) \psi_{j}}}{k \sin \psi_{j}}+\frac{\mathrm{e}^{-\mathrm{i}(2 n-1) \psi_{-j}}}{k \sin \psi_{-j}}\right]\right) \\
+\frac{2}{\pi} \sum_{j=0}^{n-1} \frac{(-1)^{j} 2^{2 j}(j+n-1) !}{(2 j+1) !(n-j-1) !} \mathrm{B}_{2 j+1}\left(\frac{\alpha}{2 \pi}\right),
\end{array}
$$

where $\mathrm{B}_{n}(\cdot)$ is a Bernoulli polynomial. The convergence of these series can easily be accelerated; see [14]. Derivatives with respect to the variable $\alpha$ are available directly from the identities

$$
\frac{\mathrm{d} \psi_{j}}{\mathrm{~d} \alpha}=-\frac{1}{k \sin \psi_{j}}
$$

and

$$
\frac{\mathrm{d}}{\mathrm{d} \alpha}\left(\frac{\mathrm{e}^{\mathrm{i} q \psi_{j}}}{k \sin \psi_{j}}\right)=\frac{\mathrm{e}^{\mathrm{i} q \psi_{j}}}{k^{2} \sin ^{3} \psi_{j}}\left(\cos \psi_{j}-\mathrm{i} q \sin \psi_{j}\right),
$$

which hold for all values of $j$ and $q$. Finally, from equation (16), we note that $\psi_{j}=0$ and $\psi_{j}=\pi$ correspond to the points

$$
\alpha=k-2 j \pi
$$

and

$$
\alpha=-k-2 j \pi
$$

$j \in \mathbb{Z}$, respectively. It now follows from (17) that these are order one (square root) branch points of the function $\sigma_{n}(\alpha)$. 


\section{References}

[1] P. McIver, C. M. Linton, and M. McIver. Construction of trapped modes for wave guides and diffraction gratings. Proc. Roy. Soc. Lond., A, 454:2593-2616, 1998.

[2] R. Porter and D. V. Evans. Rayleigh-Bloch surface waves along periodic gratings and their connection with trapped modes in waveguides. J. Fluid Mech., 386:233-258, 1999.

[3] C. M. Linton and M. McIver. The existence of Rayleigh-Bloch surface waves. J. Fluid Mech., 470:85-90, 2002.

[4] Anne-Sophie Bonnet-Bendhia and Felipe Starling. Guided waves by electromagnetic gratings and non-uniqueness examples for the diffraction problem. Math. Meth. in the Appl. Sci., 17:305-338, 1994.

[5] R. A. Sigelmann and A. Ishimaru. Radiation from periodic structures excited by an aperiodic source. IEEE Trans. Antennas Propagat., 13:354-364, 1965.

[6] R. J. Mailloux. Excitation of a surface wave along an infinite Yagi-Uda array. IEEE Trans. Antennas Propagat., 13:719-724, 1965.

[7] Hung-Yu David Yang and David R. Jackson. Theory of line-source radiation from a metal-strip grating dielectric-slab structure. IEEE Trans. Antennas Propagat., 48:556$564,2000$.

[8] Filippo Capolino, David R. Jackson, and Donald R. Wilton. Fundamental properties of the field at the interface between air and a periodic artificial material excited by a line source. IEEE Trans. Antennas Propagat., 53:91-99, 2005.

[9] Filippo Capolino, David R. Jackson, and Donald R. Wilton. Mode excitation from sources in two-dimensional EBG waveguides using the array scanning method. IEEE Microwave Theory and Wireless Component Letters, 15:49-51, 2005.

[10] C. M. Linton and P. McIver. Handbook of Mathematical Techniques for Wave/Structure Interactions. Chapman \& Hall/CRC, Boca Raton, 2001.

[11] M. Abramowitz and I. A. Stegun. Handbook of Mathematical Functions. Dover Publications, New York, 1965.

[12] C. M. Linton and D. V. Evans. The radiation and scattering of surface waves by a vertical circular cylinder in a channel. Phil. Trans. R. Soc. Lond., A, 338:325-357, 1992.

[13] V. Twersky. Elementary function representation of Schlömilch series. Arch. Rational Mech. Anal., 8:323-332, 1961.

[14] C. M. Linton. The Green's function for the two-dimensional Helmholtz equation in periodic domains. J. Engng. Math., 33:377-402, 1998. 
[15] D. V. Evans and R. Porter. Trapping and near-trapping by arrays of cylinders in waves. J. Engng. Math., 35:149-179, 1999.

[16] C. M. Linton. Schlömilch series that arise in diffraction theory and their efficient computation. J. Phys. A, 39:3325-3339, 2006.

[17] Ivar Stakgold. Green's Functions and Boundary Value Problems. John Wiley \& Sons, 2nd edition, 1998.

[18] C. M. Linton and I. Thompson. Resonant effects in scattering by periodic arrays. Submitted.

[19] D. G. Crighton, A. P. Dowling, J. E. Ffowcs Williams, M. Heckl, and F. G. Leppington. Modern Methods in Analytical Acoustics. Springer-Verlag, London, 1992. 